Mathematical Modelling AND ANALysis

Volume 18 Number 2, April 2013, 191-203

http://dx.doi.org/10.3846/13926292.2013.780189

(c) Vilnius Gediminas Technical University, 2013
Publisher: Taylor\&Francis and VGTU

http://www.tandfonline.com/TMMA

Print ISSN: 1392-6292

Online ISSN: 1648-3510

\title{
The Regions of Solvability for Some Three Point Problem
}

\section{Natalija Sergejeva}

\section{Latvia University of Agriculture}

Liela 2, LV-3001 Jelgava, Latvia

E-mail: natalijasergejeva@inbox.lv

Received September 14, 2012; revised January 14, 2013; published online April 1, 2013

Abstract. The solvability results are established for the boundary value problem $-x^{\prime \prime}=\mu x^{+}-\lambda x^{-}+h\left(t, x, x^{\prime}\right), x(0)=0, x(1)=c x\left(\frac{1}{2}\right)$, where $x^{+}=\max \{x, 0\}$, $x^{-}=\max \{-x, 0\}, h$ is a bounded nonlinearity. The existence results are based of the knowledge of the spectrum for the problem with $h \equiv 0$.

Keywords: Fučík problem, spectrum, regions of solvability.

AMS Subject Classification: 34B15.

\section{Introduction}

The classical Fučík problem is

$$
\begin{aligned}
& -x^{\prime \prime}=\mu x^{+}-\lambda x^{-}, \quad x^{+}=\max \{x, 0\}, \quad x^{-}=\max \{-x, 0\}, \\
& x(0)=0, \quad x(1)=0 .
\end{aligned}
$$

The Fučík spectrum is a set of $(\mu, \lambda)$ such that the problem (1.1), (1.2) has nontrivial solutions. This spectrum is well known [1] and some first branches of the spectrum are depicted in Figure 1.

The knowledge the Fučík spectrum is useful when we are considering the problem

$$
-x^{\prime \prime}=\mu x^{+}-\lambda x^{-}+h\left(t, x, x^{\prime}\right), \quad x(0)=0, \quad x(1)=0
$$

with a bounded right side $h$. This problem was considered in [1] and it is solvable for $(\mu, \lambda)$ from the "good" regions (see [2] also), which are shown in Figure 2.

Recently a significant attention (see $[4,8]$ and literature therein) is paid to nonlocal boundary conditions of the form $x(a)=0, x(b)=c x(\eta)$, where $\eta$ is an interior point of the considering interval $(a, b)$. We investigate the problem 


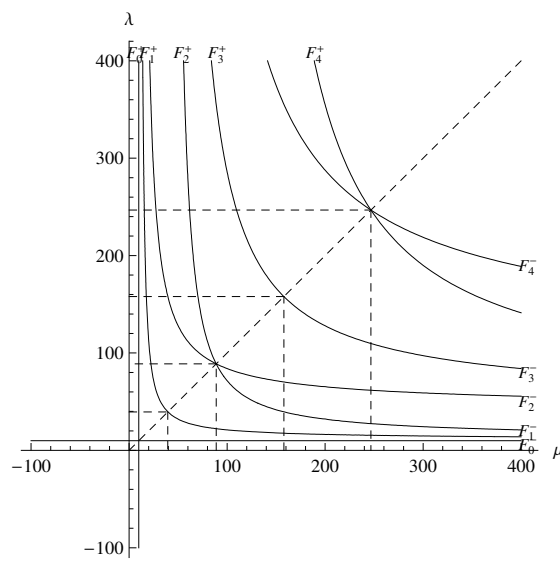

Figure 1. The spectrum of the classical Fučík problem.

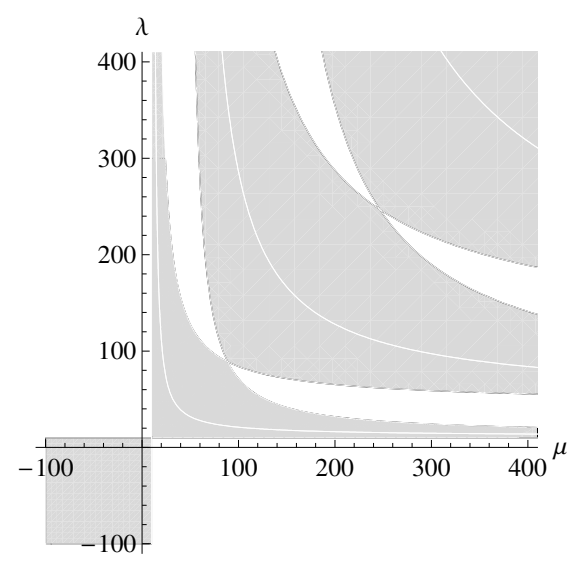

Figure 2. The solvability regions for the problem (1.3).

$$
\begin{aligned}
& -x^{\prime \prime}=\mu x^{+}-\lambda x^{-}+h\left(t, x, x^{\prime}\right), \\
& x(0)=0, \quad x(1)=c x\left(\frac{1}{2}\right), \quad c \in \mathbb{R},
\end{aligned}
$$

where $h$ is a bounded nonlinearity. To formulate existence results for the problem (1.4), (1.5) we need the precise description of the spectrum.

So first we obtain the analytical description of the spectra to the problem (1.1), (1.5) for the cases $c>0$ and $c<0$ separately. Then we study the so called "good" regions in $(\mu, \lambda)$-plane, which consist of $(\mu, \lambda)$ such that the problem (1.4), (1.5) is solvable. The existence result is formulated and proved in Section 3. Finally we provide examples for existence and non-existence of solutions.

Let us remark that usually two-index description of the branches of the classical Fučík spectrum is employed. In our notation we use three indexes for the description of some branches. This complication is justified by the following reasons. Usually only positive $\mu$ and $\lambda$ are considered. Thus the additional "." sign at the lower index in a description of a branch shows that the respective $\lambda$ or $\mu$ is negative. For instance, the notation $F_{0-}^{+}$refers to solutions of the equation $x^{\prime \prime}=-\mu x$ which do not have zeros in the interval $(0,1]$ and $\mu<0$ (these solutions are exponents, not sines and cosines). Similar two-index description of the branches was used in [7], which is devoted to the Fučík type problem with integral condition. The regions of solvability for the corresponding Fuč́k type problem with integral condition was described in $[3,6]$.

This work generalizes results known in the case of special three-point boundary condition (1.5). First results in this field (investigation the spectrum with one classical and one Bitsadze-Samarskii type nonlocal boundary condition) were given in [5]. 


\section{Some Fučík Type Problem}

Consider the problem

$$
\begin{aligned}
& -x^{\prime \prime}=\mu x^{+}-\lambda x^{-}, \\
& x(0)=0, \quad x(1)=c x\left(\frac{1}{2}\right), \quad c \in \mathbb{R} .
\end{aligned}
$$

Remark 1. If $c=0$ than the problem $(2.1),(2.2)$ reduces to the classical Fučík problem (1.1), (1.2).

The description of the spectrum of the problem $(2.1),(2.2)$ is contained in the following results.

Lemma 1. The branches $F_{0}^{ \pm}$of the spectrum for the problem (2.1), (2.2) do not exist for negative $c$ values.

Proof. To be definite, let $x^{\prime}(0)>0$. It is clear that for $c<0$ the solution of the problem must have at least one zero in the interval $(0,1)$. That is why the branches $F_{0}^{ \pm}$for negative $c$ values do not exist. The case $x^{\prime}(0)<0$ can be considered similarly.

Lemma 2. The branches $F_{0+}^{+}$and $F_{0+}^{-}$of the spectrum for the problem (2.1), (2.2) are the straight lines for $c \in[0,2]$. The branch $F_{0+}^{+}$is parallel to $(0, \lambda)$ axis and is located in the first and fourth quadrants of $(\mu, \lambda)$-plane. The branch $F_{0+}^{-}$is parallel to $(0, \mu)$ axis and is located in the first and second quadrants of $(\mu, \lambda)$-plane.

Proof. Suppose that $c>0$ and let $x(t)$ be a nontrivial solution of the problem $(2.1),(2.2)$ without zeroes in the interval $(0,1), x^{\prime}(0)>0$. The corresponding $(\mu, \lambda) \in F_{0}^{+}, \lambda \in \mathbb{R}$. It follows that the problem $(2.1),(2.2)$ reduces to the linear eigenvalue problem. Consider the $\mu>0$. We obtain $x(t)=C \sin \sqrt{\mu} t$ from the first of conditions (2.2). In view of the $x(1)=c x\left(\frac{1}{2}\right)$ we get

$$
\sin \sqrt{\mu}=c \sin \frac{\sqrt{\mu}}{2} \Longrightarrow \cos \frac{\sqrt{\mu}}{2}=\frac{c}{2} .
$$

It follows that the unique solution of the last equation which satisfies all the above considered conditions is $\mu=4\left(\arccos \frac{c}{2}\right)^{2}$ and this solution exists only for $c \in[0,2]$. This proves the lemma.

Lemma 3. The branches $F_{0-}^{+}$and $F_{0-}^{-}$of the spectrum for the problem (2.1), (2.2) are the straight lines for $c \in(2 ;+\infty)$. The branch $F_{0-}^{+}$is parallel to $(0, \lambda)$ axis and is located in the second and third quadrants. The branch $F_{0-}^{-}$is parallel to $(0, \mu)$ axis and is located in the third and fourth quadrants.

Proof. Consider the linear eigenvalue problem

$$
-x^{\prime \prime}=\mu x, \quad x(0)=0, \quad x(1)=c x\left(\frac{1}{2}\right)
$$


for $\mu<0$. The solutions of such type problems are not sine or cosine functions, but exponents. We obtain $x(t)=C \sinh \sqrt{-\mu} t$ from the first of conditions (2.2). It follows

$$
\sinh \sqrt{-\mu}=c \sinh \frac{\sqrt{-\mu}}{2} \Longrightarrow \cosh \frac{\sqrt{-\mu}}{2}=\frac{c}{2}
$$

from the conditions of the problem $(2.1),(2.2)$. The solution of the last equation is a negative number and it exists for all $c>2$. This proves the lemma.

Lemma 4. The following assertions are valid in the case of $c>0$ :

- the solutions of the problem (2.1), (2.2) which correspond to the branches $F_{4 i-3}^{ \pm}$have $2 i-1$ zeroes in the interval $\left(0, \frac{1}{2}\right)$ and $2 i-2$ zeroes in the interval $\left(\frac{1}{2}, 1\right)$;

- the solutions of the problem (2.1), (2.2) which correspond to the branches $F_{4 i-2}^{ \pm}$have $2 i-2$ zeroes in the interval $\left(0, \frac{1}{2}\right)$ and $2 i$ zeroes in the interval $\left(\frac{1}{2}, 1\right)$;

- the solutions of the problem (2.1), (2.2) which correspond to the branches $F_{4 i-1}^{ \pm}$have $2 i-1$ zeroes in the interval $\left(0, \frac{1}{2}\right)$ and $2 i$ zeroes in the interval $\left(\frac{1}{2}, 1\right)$;

- the solutions of the problem (2.1), (2.2) which correspond to the branches $F_{4 i}^{ \pm}$have the same number of zeroes on both intervals $\left(0, \frac{1}{2}\right)$ and $\left(\frac{1}{2}, 1\right)$.

Proof. The proof of Lemma can be concluded using the geometrical type arguments.

Lemma 5. The following assertions are valid in the case of $c<0$

- the solutions of the problem (2.1), (2.2) which correspond to the branches $F_{4 i-3}^{ \pm}$have $2 i-2$ zeroes in the interval $\left(0, \frac{1}{2}\right)$ and $2 i-1$ zeroes in the interval $\left(\frac{1}{2}, 1\right)$;

- the solutions of the problem (2.1), (2.2) which correspond to the $F_{4 i-2}^{ \pm}$ have the same number of zeroes on both intervals $\left(0, \frac{1}{2}\right)$ and $\left(\frac{1}{2}, 1\right)$.

Proof. The proof of Lemma can be concluded using the geometrical type arguments.

Lemma 6. The branch $F_{1}^{+}\left(F_{1}^{-}\right)$of the spectrum for the problem $(2.1),(2.2)$ exists for all $c$ values, it consists of two parts $F_{1-}^{+}, F_{1+}^{+}\left(F_{1-}^{-}, F_{1+}^{-}\right)$and a point on one of the axes for $c \in(-\infty, 0) \cup(2,+\infty)$.

Proof. To be definite, consider $F_{1}^{+}$. In view of $(\mu, \lambda) \in F_{1}^{+}$we obtain that the solution of the problem $(2.1),(2.2)$ has only one zero in the interval $(0,1)$. Let us denote it as $\tau$. It is clear that for $c \in[0,2]$ the sine function in the interval $(0, \tau)$ can be continued only with sine function in the interval $(\tau, 1)$. For other 
$c$ value the sine function in the interval $(0, \tau)$ can be continued either with sine function or with exponent or with straight line in the interval $(\tau, 1)$. In the first case we obtain the branch $F_{1+}^{+}$(it is located in the firsts quadrant), in the second one we obtain the branch $F_{1-}^{+}$(it is located in the fourth quadrant), the continuation with straight line corresponds to a point on the $\mu$-axis.

Lemma 7. The branch $F_{4 i-1}^{ \pm}$of the spectrum for the problem (2.1), (2.2) for $c \in(-\infty, 0)$ coincide with the respective branch $F_{4 i-1}^{ \pm}$of the spectrum for the classical Fučik problem (1.1), (1.2).

Proof. To be definite, consider $F_{3}^{+}$. The corresponding nontrivial solution $x(t)$ of the problem (2.1), (2.2) has three zeroes in the interval $(0,1)$. For negative $c$ values $x\left(\frac{1}{2}\right)$ and $x(1)$ have different signs. This can be performed in three cases. First, all three zeroes are located in the interval $\left(\frac{1}{2}, 1\right)$. It is clear that it is impossible. Second, two of zeroes are in the interval $\left(0, \frac{1}{2}\right)$ and the third of them is located in the interval $\left(\frac{1}{2}, 1\right)$. In view of location of zeroes we obtain

$$
\left\{\begin{array}{l}
\pi / \sqrt{\mu}+\pi / \sqrt{\lambda}<\frac{1}{2} \\
2 \pi / \sqrt{\mu}+\pi / \sqrt{\lambda}<1 \\
2 \pi / \sqrt{\mu}+2 \pi / \sqrt{\lambda}>1 .
\end{array}\right.
$$

The first and third inequalities contradict each other. It follows that such location of zeroes is impossible also.

Third, the second zero $\tau_{2}=\frac{1}{2}$. In view of it

$$
\frac{\pi}{\sqrt{\mu}}+\frac{\pi}{\sqrt{\lambda}}=\frac{1}{2} \quad \Longrightarrow \quad \frac{2 \pi}{\sqrt{\mu}}+\frac{2 \pi}{\sqrt{\lambda}}=1 .
$$

The corresponding $(\mu, \lambda)$ values form the branch $F_{3}^{+}$of the spectrum for the classical Fučík problem (1.1), (1.2) [1]. The proof for other $i$ values is similar.

Lemma 8. The branch $F_{4 i}^{ \pm}$of the spectrum for the problem (2.1), (2.2) does not exist for $c \in(-\infty, 0)$.

Proof. To be definite, consider the branch $F_{4}^{+}$. Suppose the contrary that the solutions with four zeroes in the interval $(0,1)$ exist for negative $c$ values. For such $c$ values $x\left(\frac{1}{2}\right)$ and $x(1)$ have different signs. This can be performed in two cases. First, the first zero is in the interval $\left(0, \frac{1}{2}\right)$ and the other are in the interval $\left(\frac{1}{2}, 1\right)$. It follows

$$
\left\{\begin{array}{l}
\pi / \sqrt{\mu}<\frac{1}{2} \\
\pi / \sqrt{\mu}+\pi / \sqrt{\lambda}>\frac{1}{2} \\
2 \pi / \sqrt{\mu}+2 \pi / \sqrt{\lambda}<1 \\
3 \pi / \sqrt{\mu}+2 \pi / \sqrt{\lambda}>1
\end{array}\right.
$$

Thus, the second and third inequalities contradict each other. 
Second, three zeroes in the interval $\left(0, \frac{1}{2}\right)$ and the last of them is located in the interval $\left(\frac{1}{2}, 1\right)$. We obtain

$$
\left\{\begin{array}{l}
2 \pi / \sqrt{\mu}+\pi / \sqrt{\lambda}<\frac{1}{2} \\
2 \pi / \sqrt{\mu}+2 \pi / \sqrt{\lambda}>\frac{1}{2} \\
2 \pi / \sqrt{\mu}+2 \pi / \sqrt{\lambda}<1 \\
3 \pi / \sqrt{\mu}+2 \pi / \sqrt{\lambda}>1
\end{array}\right.
$$

The first and fourth inequalities contradict each other. The obtained contradictions prove the lemma.

Theorem 1. The spectrum of the problem (2.1), (2.2) for $c \geq 0$ consists of the branches (if these branches exist) given by (where $i=1,2, \ldots$ )

$$
\begin{aligned}
& F_{0}^{+}=\left\{(\mu, \lambda) \mid \sin \sqrt{\mu}=c \sin \frac{\sqrt{\mu}}{2}, \mu \leq \pi^{2}, \lambda \in \mathbb{R} \text { for } c \in[0,2] ;\right. \\
& \left.\sinh \sqrt{-\mu}=c \sinh \frac{\sqrt{-\mu}}{2}, \mu<0, \lambda \in \mathbb{R} \text { for } c \in(2 ;+\infty)\right\}, \\
& F_{1}^{+}=F_{1+}^{+} \cup F_{1-}^{+} \text {, } \\
& F_{1-}^{+}=\left\{(\mu, \lambda) \mid \sinh \left(\sqrt{-\lambda}-\pi \sqrt{-\frac{\lambda}{\mu}}\right)=c \sinh \left(\frac{-\sqrt{\lambda}}{2}-\pi \sqrt{-\frac{\lambda}{\mu}}\right),\right. \\
& \left.\mu>\pi^{2}, \lambda<0\right\} \text {, } \\
& F_{1+}^{+}=\left\{(\mu, \lambda) \mid \sin \left(\sqrt{\lambda}-\pi \sqrt{\frac{\lambda}{\mu}}\right)=c \sin \left(\frac{\sqrt{\lambda}}{2}-\pi \sqrt{\frac{\lambda}{\mu}}\right)\right. \text {, } \\
& \left.\mu>\pi^{2}, \pi / \sqrt{\mu}+\pi / \sqrt{\lambda} \geq 1\right\}, \\
& F_{4 i-3}^{+}=\left\{(\mu, \lambda) \mid \sin \left(\sqrt{\lambda}-\pi(2 i-1) \sqrt{\frac{\lambda}{\mu}}\right)=c \sin \left(\frac{\sqrt{\lambda}}{2}-\pi i \sqrt{\frac{\lambda}{\mu}}-\pi(i-1)\right),\right. \\
& (2 i-1) \pi / \sqrt{\mu}+(2 i-2) \pi / \sqrt{\lambda}<1,(2 i-1) \pi / \sqrt{\mu}+(2 i-1) \pi / \sqrt{\lambda} \geq 1\}, \\
& F_{4 i-2}^{+}=\left\{(\mu, \lambda) \mid \sin \left(\sqrt{\mu}-\pi(2 i-1) \sqrt{\frac{\mu}{\lambda}}\right)=-c \sin \left(\frac{\sqrt{\mu}}{2}-\pi(i-1) \sqrt{\frac{\mu}{\lambda}}\right.\right. \\
& \left.-\pi(i-1)), \frac{(2 i-1) \pi}{\sqrt{\mu}}+\frac{(2 i-1) \pi}{\sqrt{\lambda}}<1, \frac{2 i \pi}{\sqrt{\mu}}+\frac{(2 i-1) \pi}{\sqrt{\lambda}} \geq 1\right\}, \\
& F_{4 i-1}^{+}=\left\{(\mu, \lambda) \mid \sin \left(\sqrt{\lambda}-2 \pi i \sqrt{\frac{\lambda}{\mu}}\right)=-c \sin \left(\frac{\sqrt{\lambda}}{2}-\pi i \sqrt{\frac{\lambda}{\mu}}-\pi(i-1)\right)\right. \text {, } \\
& 2 \pi i / \sqrt{\mu}+(2 i-1) \pi / \sqrt{\lambda}<1,2 \pi i / \sqrt{\mu}+2 \pi i / \sqrt{\lambda} \geq 1\}, \\
& F_{4 i}^{+}=\left\{(\mu, \lambda) \mid \sin \left(\sqrt{\mu}-2 \pi i \sqrt{\frac{\mu}{\lambda}}\right)=c \sin \left(\frac{\sqrt{\mu}}{2}-\pi i \sqrt{\frac{\mu}{\lambda}}-\pi i\right),\right. \\
& 2 \pi i / \sqrt{\mu}+2 \pi i / \sqrt{\lambda}<1,(2 i+1) \pi / \sqrt{\mu}+2 \pi i / \sqrt{\lambda} \geq 1\}, \\
& F_{i}^{-}=\left\{(\mu, \lambda) \mid(\lambda, \mu) \in F_{i}^{+}\right\} .
\end{aligned}
$$

Remark 2. $F_{1+}^{+}=F_{4 i-3}^{+}$if $i=1$.

Proof. Consider the problem (2.1), (2.2) with $c>0$. The proof of expressions for $F_{0}^{ \pm}$follows from Lemmas 1-3. The ideas of the proof for all branches are 
similar. We consider the eigenvalue problems in the intervals between two consecutive zeroes of the solution in view of the above results and use the conditions of the solutions for these problems.

For example we will prove the theorem for the $F_{2}^{+}$. The corresponding solution has two zeroes. Let us denote these zeroes by $\tau_{1}$ and $\tau_{2}$. Consider a solution of the problem $(2.1),(2.2)$ in the interval $\left(0, \tau_{1}\right)$. We obtain that the problem in this interval reduces to the linear eigenvalue problem

$$
-x^{\prime \prime}=\mu x, \quad x(0)=x\left(\tau_{1}\right)=0 .
$$

The corresponding solution is

$$
x(t)=A \sin (\sqrt{\mu} t), \quad A>0 .
$$

Therefore $\tau_{1}=\frac{\pi}{\sqrt{\mu}}$.

Now consider a solution of the problem (2.1), (2.2) in the interval $\left(\tau_{1}, \tau_{2}\right)$. We obtain the eigenvalue problem

$$
-x^{\prime \prime}=\lambda x, \quad x\left(\tau_{1}\right)=x\left(\tau_{2}\right)=0, \quad x^{\prime}\left(\tau_{1}\right)<0 .
$$

From the last boundary condition we must determine also $\tau_{2}$. The solution of this problem is the function

$$
x(t)=-B \sin \left(\sqrt{\lambda}\left(t-\tau_{1}\right)\right), \quad B>0, \quad \tau_{2}=\frac{\pi}{\sqrt{\mu}}+\frac{\pi}{\sqrt{\lambda}} .
$$

Similarly a solution of the problem $(2.1),(2.2)$ in the interval $\left(\tau_{2}, 1\right)$ is

$$
x(t)=A \sin \left(\sqrt{\mu}\left(t-\tau_{2}\right)\right) .
$$

From Lemma 4 we obtain that both zeroes of the solution for the problem $(2.1),(2.2)$ are located in the interval $\left(\frac{1}{2}, 1\right)$. In view of the condition $x(1)=$ $c x\left(\frac{1}{2}\right)$ and $(2.3),(2.5)$ we obtain

$$
A \sin \left(\sqrt{\mu}\left(1-\left(\frac{\pi}{\sqrt{\mu}}+\frac{\pi}{\sqrt{\lambda}}\right)\right)\right)=c A \sin \left(\frac{\sqrt{\mu}}{2}\right)
$$

or

$$
\sin \left(\sqrt{\mu}-\pi \sqrt{\frac{\mu}{\lambda}}\right)=-c \sin \left(\frac{\sqrt{\mu}}{2}\right) .
$$

In view of the structure of solution which corresponds to $F_{2}^{+}$we obtain

$$
\frac{\pi}{\sqrt{\mu}}+\frac{\pi}{\sqrt{\lambda}}<1 \leq \frac{2 \pi}{\sqrt{\mu}}+\frac{\pi}{\sqrt{\lambda}} .
$$

This result and (2.6) prove the theorem for $F_{2}^{+}$. The proof for other branches is similar.

Theorem 2. The spectrum of the problem (2.1), (2.2) for $c<0$ consists of the branches (if any exist) given by (where $i=1,2, \ldots$ )

$$
F_{1}^{+}=F_{1+}^{+} \cup F_{1-}^{+},
$$




$$
\begin{aligned}
F_{1-}^{+}= & \left\{(\mu, \lambda) \mid \sinh \left(\sqrt{-\lambda}-\pi \sqrt{-\frac{\lambda}{\mu}}\right)=-c \sqrt{-\frac{\lambda}{\mu}} \sinh \left(\frac{\sqrt{\mu}}{2}\right), \mu>\pi^{2}, \lambda<0\right\}, \\
F_{1+}^{+}= & \left\{(\mu, \lambda) \mid \sin \left(\sqrt{\lambda}-\pi \sqrt{\frac{\lambda}{\mu}}\right)=-c \sqrt{\frac{\lambda}{\mu}} \sin \left(\frac{\sqrt{\mu}}{2}\right), \mu>\pi^{2}, \frac{\pi}{\sqrt{\mu}}+\frac{\pi}{\sqrt{\lambda}} \geq 1\right\}, \\
F_{4 i-3}^{+}= & \left\{(\mu, \lambda) \mid \sin \left(\sqrt{\lambda}-\pi(2 i-1) \sqrt{\frac{\lambda}{\mu}}\right)=-c \sqrt{\frac{\lambda}{\mu}} \sin \left(\frac{\sqrt{\mu}}{2}-\pi(i-1) \sqrt{\frac{\mu}{\lambda}}\right.\right. \\
& \left.-\pi(i-1)), \frac{(2 i-1) \pi}{\sqrt{\mu}}+\frac{(2 i-2) \pi}{\sqrt{\lambda}}<1, \frac{(2 i-1) \pi}{\sqrt{\mu}}+\frac{(2 i-1) \pi}{\sqrt{\lambda}} \geq 1\right\}, \\
F_{4 i-2}^{+}= & \left\{(\mu, \lambda) \mid \sin \left(\sqrt{\mu}-\pi(2 i-1) \sqrt{\frac{\mu}{\lambda}}\right)=c \sqrt{\frac{\mu}{\lambda}} \sin \left(\frac{\sqrt{\lambda}}{2}-\pi i \sqrt{\frac{\lambda}{\mu}}\right.\right. \\
& \left.-\pi(i-1)), \frac{(2 i-1) \pi}{\sqrt{\mu}}+\frac{(2 i-1) \pi}{\sqrt{\lambda}}<1, \frac{2 i \pi}{\sqrt{\mu}}+\frac{(2 i-1) \pi}{\sqrt{\lambda}} \geq 1\right\}, \\
F_{4 i-1}^{+}= & \left\{(\mu, \lambda) \mid \frac{2 \pi i}{\sqrt{\mu}}+\frac{2 \pi i}{\sqrt{\lambda}}=1\right\}, \quad F_{i}^{-}=\left\{(\mu, \lambda) \mid(\lambda, \mu) \in F_{i}^{+}\right\} .
\end{aligned}
$$

Proof. The proof of this theorem is similar to the proof of Theorem 1 taking into account Lemmas 1 and 5-8.

Remark 3. The branches of the spectrum for the problem (2.1), (2.2) are bounded by the respective branches of the classical Fučík spectrum. For example the branch $F_{2}^{+}$related to $(2.1)$, (2.2)is located in the region between the branches $\mathcal{F}_{1}^{+}$and $\mathcal{F}_{2}^{+}$related to the classical Fučík spectrum.

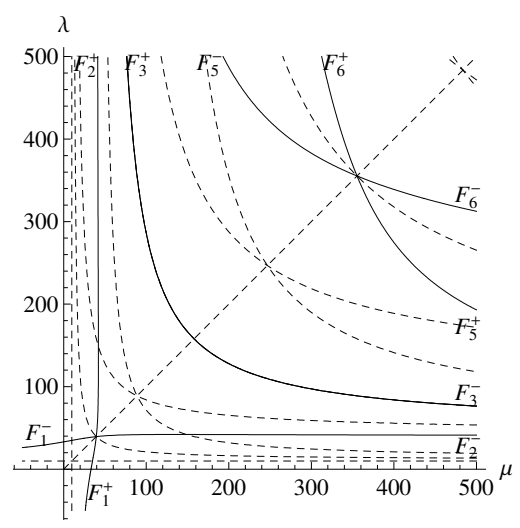

Figure 3. Some spectrum branches for $c=-10$.

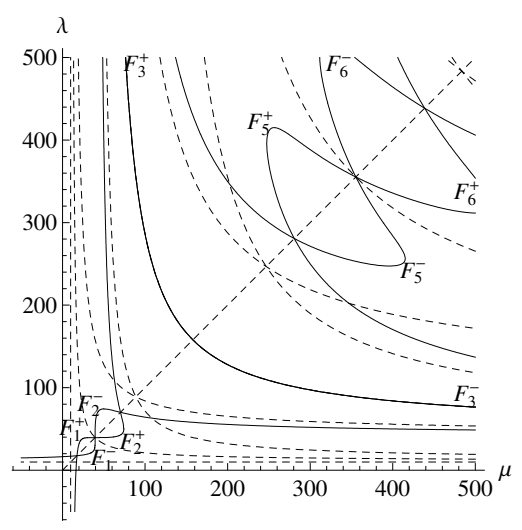

Figure 4. Some spectrum branches for $c=-1$.

Some branches of the spectrum for the problem (2.1), (2.2) are depicted in Figures 3-6 for different $c$ values (the Fučík spectrum branches are shown by dashed curves).

\section{The BVP with a Nonlinearity in the Right Side}

Consider the problem (1.4), (1.5), where $h$ is a bounded continuous function. 


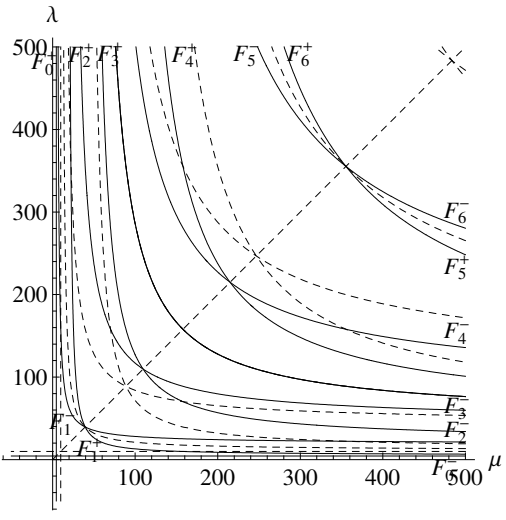

Figure 5. Some spectrum branches for $c=1$.

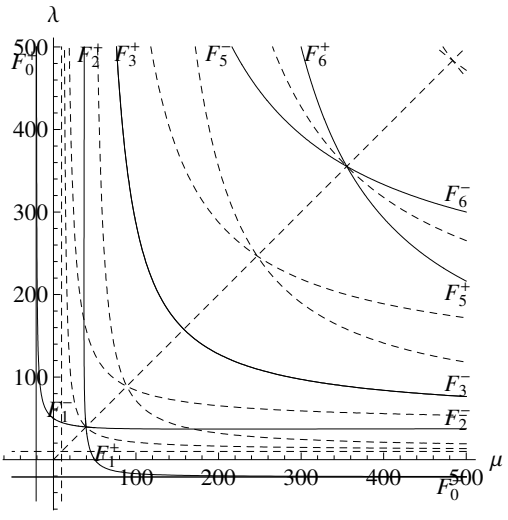

Figure 6. Some spectrum branches for $c=10$.

Analogously as for Dirichlet problem (see $[1,2]$ ) the knowledge of the spectrum of the problem (2.1), (2.2) can be used to define regions of solvability for the problem (1.4), (1.5).

We assume that $h$ is such that solutions of (1.4) continuously depend on the right sides of an equation (the Lipschitz condition with respect to $x$ and $x^{\prime}$ is sufficient). Consider the Cauchy problem

$$
-x^{\prime \prime}=\mu x^{+}-\lambda x^{-}+h\left(t, x, x^{\prime}\right), \quad x(0)=0, \quad x^{\prime}(0)=\alpha .
$$

Let $x(t ; \alpha)$ be a solution of (3.1). Consider also the Cauchy problems

$$
-z^{\prime \prime}=\mu z^{+}-\lambda z^{-}, \quad z(0)=0, \quad z^{\prime}(0)=1
$$

and

$$
-z^{\prime \prime}=\mu z^{+}-\lambda z^{-}, \quad z(0)=0, \quad z^{\prime}(0)=-1 .
$$

Let functions $z_{+}(t)$ and $z_{-}(t)$ be solutions of the Cauchy problems (3.2) and (3.3) respectively.

Lemma 9. The functions $u(t ; \alpha)=\frac{1}{\alpha} x(t ; \alpha)$ tend uniformly in $t \in[0,1]$ to the function $z_{+}(t)$ as $\alpha \rightarrow+\infty$ and to $z_{-}(t)$ as $\alpha \rightarrow-\infty$.

Proof. The functions $u(t ; \alpha)$ satisfy the problem

$$
-u^{\prime \prime}=\mu u^{+}-\lambda u^{-}+\frac{1}{\alpha} h\left(t, x(t), x^{\prime}(t)\right), \quad u(0)=0, \quad u^{\prime}(0)=1 .
$$

The last term $\left(\frac{1}{\alpha} h\right)$ in the above equation tends to zero as $\alpha \rightarrow \infty$ since $h$ is bounded. Therefore, by continuous dependence of solutions on the right sides of an equation, $u(t ; \alpha)$ tend uniformly in $t \in[0,1]$ to $z_{+}(t)$ as $\alpha \rightarrow+\infty$ and to $z_{-}(t)$ as $\alpha \rightarrow-\infty$.

The following result is valid. 
Theorem 3. If

$$
\left(z_{+}(1)-c z_{+}\left(\frac{1}{2}\right)\right)\left(z_{-}(1)-c z_{-}\left(\frac{1}{2}\right)\right)<0,
$$

where meaning of $z_{+}(t)$ and $z_{-}(t)$ and restrictions on $h\left(t, x, x^{\prime}\right)$ are given above, then there exists $\alpha_{0} \in \mathbb{R}$ such that $x\left(t ; \alpha_{0}\right)$ solves the problem (1.4), (1.5).

Proof. The proof of this theorem follows from Lemma 9.

We have obtained that the problem (1.4), (1.5) is solvable if $(\mu, \lambda)$ is not in the spectrum of the problem (2.1), (2.2) but it is such that (3.4) holds ("good" regions for solvability).

The regions of $(\mu, \lambda)$-plane where (3.4) holds for some $c$ values are shown in Figures 7 and 8.
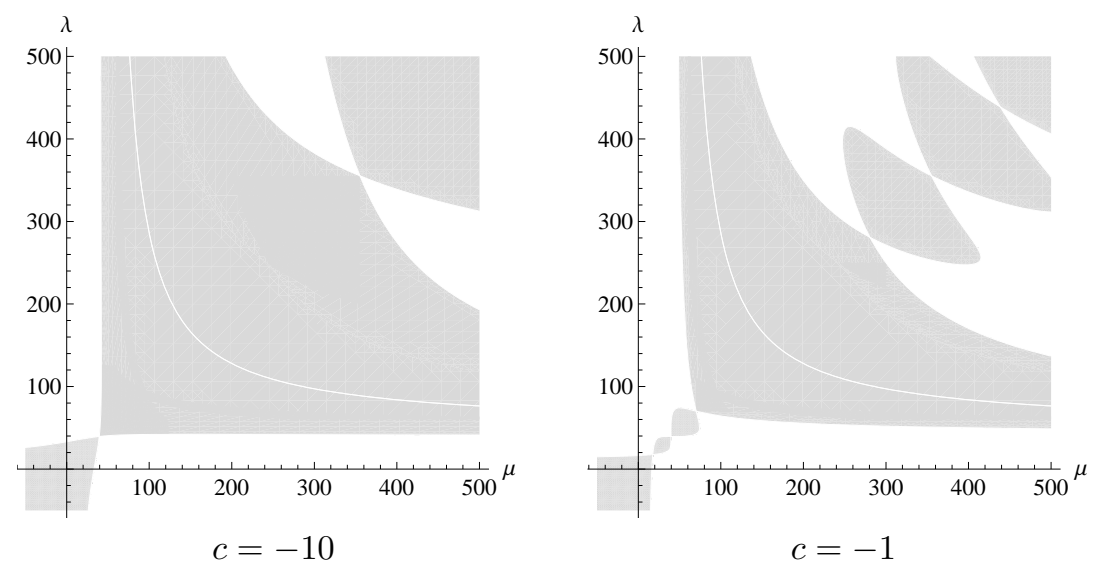

Figure 7. The solvability regions for $c<0$.
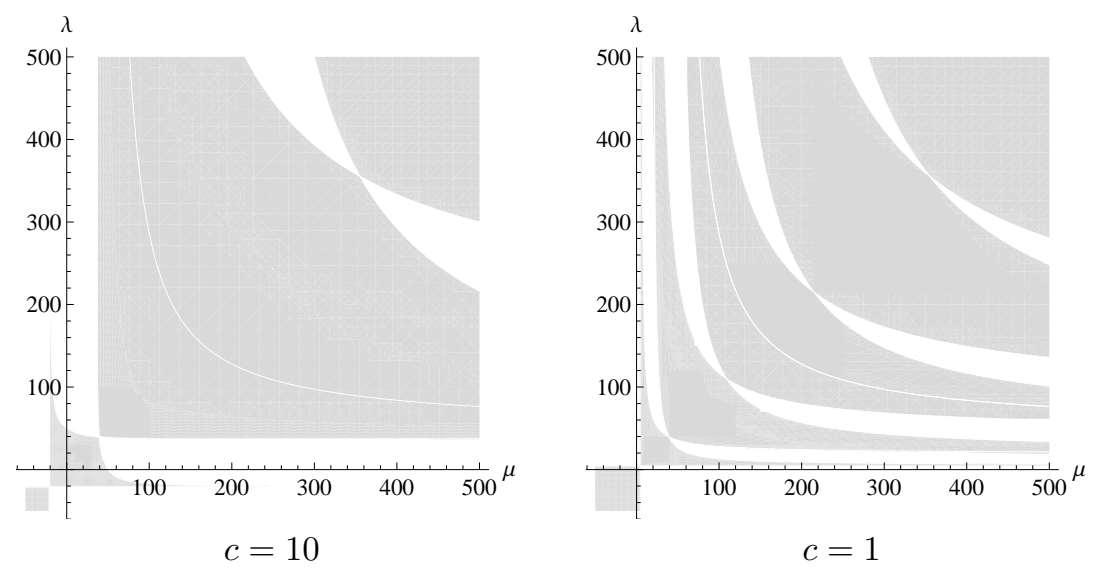

Figure 8. The solvability regions for $c>0$. 


\section{Examples}

Example 1. Consider the problem

$$
-x^{\prime \prime}=25 x^{+}+h(t), \quad \text { where } h(t)= \begin{cases}0, & \text { if } t \in\left[0, \frac{\pi}{5}\right] \\ t-\frac{\pi}{5}, & \text { if } t \in\left(\frac{\pi}{5}, 1\right]\end{cases}
$$

with conditions

$$
x(0)=0, \quad x(1)=10 x\left(\frac{1}{2}\right) .
$$

Consider the Cauchy problems

$$
-z^{\prime \prime}=25 z^{+}, \quad z(0)=0, \quad z^{\prime}(0)=1
$$

and

$$
-z^{\prime \prime}=25 z^{+}, \quad z(0)=0, \quad z^{\prime}(0)=-1 .
$$

The function

$$
z_{+}(t)= \begin{cases}\frac{1}{5} \sin 5 t, & \text { if } t \in\left[0, \frac{\pi}{5}\right] \\ -t+\frac{\pi}{5}, & \text { if } t \in\left(\frac{\pi}{5}, 1\right]\end{cases}
$$

is a solution of the problem (4.3). It follows

$$
z_{+}(1)-10 z_{+}\left(\frac{1}{2}\right)=-1+\frac{\pi}{5}-10 \cdot \frac{1}{5} \sin \frac{5}{2}=-1+\frac{\pi}{5}-2 \sin \frac{5}{2}<0 .
$$

The function $z_{-}(t)=-t$ is a solution of the problem (4.4). In view of it

$$
z_{-}(1)-10 z_{-}\left(\frac{1}{2}\right)=-1-10 \cdot\left(-\frac{1}{2}\right)=4>0 .
$$

Therefore the condition (3.4) holds and the problem (4.1), (4.2) is solvable $((\mu, \lambda)=(25,0)$ belongs to the "good" region $)$.

Remark 4. The construction of the function $h$ allows to get an explicit formula of solution for the problem (4.1), (4.2) and to construct it (see Figure 9). So

$$
x(t)= \begin{cases}-\alpha t, & \text { if } t \in\left[0, \frac{\pi}{5}\right], \\ -\frac{t^{3}}{6}+\frac{\pi t^{2}}{10}-\left(\alpha+\frac{\pi^{2}}{50}\right) t+\frac{\pi^{3}}{750}, & \text { if } t \in\left(\frac{\pi}{5}, 1\right],\end{cases}
$$

where $\alpha=x^{\prime}(0)=\frac{1}{3000}\left(125-75 \pi+15 \pi^{2}-\pi^{3}\right) \approx 0.00214$.

Example 2. Consider the problem

$$
-x^{\prime \prime}=\mu x^{+}+h(t), \quad \text { where } h(t)= \begin{cases}0, & \text { if } t \in\left[0, \frac{\pi}{\sqrt{\mu}}\right], \\ t-\frac{\pi}{\sqrt{\mu}}, & \text { if } t \in\left(\frac{\pi}{\sqrt{\mu}}, 1\right]\end{cases}
$$

for $\mu>4 \pi^{2}$ with conditions

$$
x(0)=0, \quad x(1)=-10 x\left(\frac{1}{2}\right) .
$$

Consider the Cauchy problem

$$
-z^{\prime \prime}=\mu z^{+}, \quad z(0)=0, \quad z^{\prime}(0)=1
$$




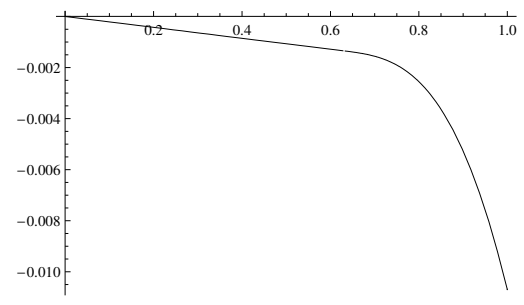

Figure 9. Graph of the solution for the problem (4.1), (4.2).

and

$$
-z^{\prime \prime}=\mu z^{+}, \quad z(0)=0, \quad z^{\prime}(0)=-1 .
$$

The function

$$
z_{+}(t)= \begin{cases}\frac{1}{\sqrt{\mu}} \sin \sqrt{\mu} t, & \text { if } t \in\left[0, \frac{\pi}{\sqrt{\mu}}\right], \\ -t+\frac{\pi}{\sqrt{\mu}}, & \text { if } t \in\left(\frac{\pi}{\sqrt{\mu}}, 1\right],\end{cases}
$$

is a solution of the problem (4.7). It follows

$$
z_{+}(1)+10 z_{+}\left(\frac{1}{2}\right)=-1+\frac{\pi}{\sqrt{\mu}}+10 \cdot\left(-1+\frac{\pi}{\sqrt{\mu}}\right)=-11+\frac{11 \pi}{\sqrt{\mu}}<0 .
$$

The function $z_{-}(t)=-t$ is a solution of the problem (4.8). In view of it

$$
z_{-}(1)+10 z_{-}\left(\frac{1}{2}\right)=-1+10 \cdot\left(-\frac{1}{2}\right)=-6<0 .
$$

Therefore the condition (3.4) does not hold. That is why we can not guarantee the solvability of the problem $(4.5),(4.6)\left((\mu, 0)\right.$ with $\mu>4 \pi^{2}$ does not belong to the "good" region).

Remark 5. Similarly as in last remark the construction of the function $h$ allows to get an explicit formulas for the problem (4.5), (4.6). The calculations show that there does not exist $x^{\prime}(0)=\alpha_{0}$ such that $x\left(t, \alpha_{0}\right)$ solves the problem $(4.5)$, (4.6).

Let us try to construct a solution of the problem (4.5), (4.6) for $x^{\prime}(0)=\alpha>$ 0 , for $\alpha<0$ and for $\alpha=0$. In the first case a solution has a zero at $\frac{\pi}{\sqrt{\mu}}<\frac{1}{2}$, then the equation $-x^{\prime \prime}=t-\frac{\pi}{\sqrt{\mu}}$ acts. It appears that then $\alpha$ must be negative. It follows that a solution with $x^{\prime}(0)>0$ does not exist. The other two cases are easier and a solution does not exist in these case also. The condition $\mu>4 \pi^{2}$ follows from $\frac{\pi}{\sqrt{\mu}}<\frac{1}{2}$.

\section{Conclusions}

The following results were obtained:

- analytical description of the spectrum for the problem (2.1), (2.2) is given;

- visualization of the spectrum for the problem (2.1), (2.2) was obtained for some values of $c$; 
- some features of the above spectrum is described for both positive and negative values of $c$;

- the problem (1.4), (1.5) was considered and the existence of solutions was established by making use of previously studied spectra for the Fučík equation;

- the examples for existence and non-existence of the solutions for the problem (1.4), (1.5) were provided.

\section{Acknowledgements}

The author would like to thank Prof. Felix Sadyrbaev for his valuable comments.

Publication and dissemination of research results has been made due to the funding of the ERAF Project Promotion of scientific activities of LUA, Contract Nr. 2010/0198/2DP/2.1.1.2.0/10/APIA/VIAA/020.

\section{References}

[1] S. Fučík and A. Kufner. Nonlinear Differential Equations. Elsevier, 1980.

[2] A. Gritsans and F. Sadyrbaev. Nonlinear problems with asymmetric principal part. Math. Model. Anal., 17(2):217-226, 2012. http://dx.doi.org/10.3846/13926292.2012.661697.

[3] A. Gritsans, F. Sadyrbaev and N. Sergejeva. Two parameter nonlinear eigenvalue problems. In A. Cabada, E. Liz and J. J. Nieto(Eds.), Mathematical Models in Engineering, Biology, and Medicine: Proceedings of the International Conference on Boundary Value Problems, volume 1124 of American Institute of Physics Conference Proceedings, pp. 185-194, Melville, New York, 2009. AIP.

[4] S. K. Ntouyas. Nonlocal initial and boundary value problems: a survey. In A. Cabada, P. Drábek and A. Fonda(Eds.), Handbook of Differential Equations, volume 2 of Ordinary Differential Equations, pp. 461-558, North Holland, 2005. Elsevier.

[5] M. P. Sapagovas and A. D. Stikonas. On the structure of the spectrum of a differential operator with a nonlocal condition. Differ. Equ., 41(7):1010-1018, 2005. http://dx.doi.org/10.1007/s10625-005-0242-y.

[6] N. Sergejeva. Fučík spectrum for the second order BVP with nonlocal boundary condition. Nonlinear Anal. Model. Control, 12(3):419-429, 2007.

[7] N. Sergejeva. On some problems with nonlocal integral condition. Math. Model. Anal., 15(1):113-126, 2010. http://dx.doi.org/10.3846/1392-6292.2010.15.113-126.

[8] J. R. L. Webb and G. Infante. Non-local boundary value problems of arbitrary order. J. London Math. Soc., 79(1):238-258, 2009.

http://dx.doi.org/10.1112/jlms/jdn066. 